\title{
An introduction to the special issue "Small Renal Masses (SRMs): update in diagnosis, management and new ablative modalities"
}

\author{
Antonio Celia ${ }^{1}$, Richard Naspro ${ }^{2}$ \\ 'Urology Unit, San Bassiano Hospital, Bassano del Grappa 36061, Italy. \\ ${ }^{2}$ UOC Urologia, ASST Papa Giovanni XXIII, Bergamo, Italy.
}

Correspondence to: Dr. Antonio Celia, Chief of Urology Unit, San Bassiano Hospital, Bassano del Grappa (VI) 36061, Italy. E-mail: antoniocel70@yahoo.it

How to cite this article: Celia A, Naspro R. An introduction to the special issue "Small Renal Masses (SRMs): update in diagnosis, management and new ablative modalities". Mini-invasive Surg 2020;4:5. http://dx.doi.org/10.20517/2574-1225.2018.007

Received: 1 Dec 2018 Accepted: 15 Jan 2020 Published: 20 Jan 2020

Science Editor: Antonio Celia Copy Editor: Jing-Wen Zhang Production Editor: Jing Yu

Renal cell carcinoma (RCC) is the most common primary malignancy of the kidney and accounts for almost $2 \%$ of all cancers. Approximately 270,000 new RCC cases are diagnosed worldwide each year. The highest incidence of RCC is reported in Western countries, with 100,000 new cases per year in Europe. Over the last decades an increase in the detection of localized RCC has been observed, probably due to the widespread use of sectional imaging accounting for incidental diagnosis ${ }^{[1]}$.

In the 1980s, only $12 \%$ of RCC cases were diagnosed as stage T1a and more than $60 \%$ accounted for stages T3-4. Currently, almost $60 \%$ are stage T1a at diagnosis and locally advanced or primary metastatic renal tumours account for only a quarter of all incidental cases ${ }^{[2]}$.

The highest incidence of localized tumours or Small Renal Masses (SRMs) is found in the elderly patients, who typically present with a high number of comorbidities. As approximately $70 \%-90 \%$ of these SRMs are malignant RCC, treatment may be required. This has certainly generated great interest in delivering better cancer care for older, more complex patients in a more tailored fashion.

Surgery still represents the standard of care for localized renal cancer. Partial nephrectomy, being open, laparoscopic or robotic has emerged as the treatment of choice for stage T1a-b tumours. Even in the presence of larger tumours, organ preservation can be considered when technically feasible and in select

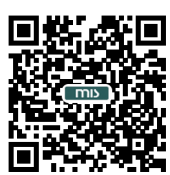


patients. The advantages of a nephron-sparing approach are clearly related to renal function preservation guaranteeing consistent oncological outcomes.

Although radical surgical procedures remain the definitive recommended treatment of SRM, non-surgical management or ablative techniques have emerged recently, particularly for smaller tumours $(<4 \mathrm{~cm})$ and for those patients who are not eligible for surgery. Although the overall oncological outcomes are still under evaluation, ablative techniques could theoretically offer the benefit of nephron-sparing treatment with the clear advantages of minimally invasive approaches.

Ablative techniques include cryoablation, radiofrequency ablation, microwave ablation, laser thermal ablation and high-intensity focused ultrasound. Evidence from the literature is more extensive for cryoablation and radiofrequency ablation while the other modalities are still to be considered experimental ${ }^{[3]}$.

To date, appropriate selection of the best therapeutic option needs to be determined on a case by case basis with thorough patient counseling. There is always a need to find the right balance between the benefits of a given treatment and its risks, without forgetting patients' characteristics.

In this context, ablative modalities seem to be a potentially valid treatment option that can reduce the morbidity and complications related with surgical procedures with acceptable oncologic and functional outcomes.

However, considering the literature, only few series are reporting intermediate - long term survival data and several studies are still evaluating the oncologic efficacy of ablative modalities.

The overall low evidence found in the literature and the lack of standardized techniques are still to be considered as major limitations for these non surgical approaches. Multicentric, randomized high volume trials are typically very complicated to perform in these settings. However, higher quality data from larger series coming from expert centres, focusing on standardization and safety are eagerly awaited for in order to obtain better and comparable oncological outcomes and to allow better reproducibility and teaching of the techniques.

The aim of this review is to focus on the best evidence available on the overall management of SRM highlighting the process from the diagnosis to the non surgical treatment modalities.

\section{DECLARATIONS}

\section{Authors' contributions}

Wrote and reviewed the manuscript: Celia A, Naspro R

\section{Availability of data and materials}

Not applicable.

\section{Financial support and sponsorship}

None.

\section{Conflicts of interest}

Both authors declared that there are no conflicts of interest. 


\section{Ethical approval and consent to participate}

Not applicable.

\section{Consent for publication}

Not applicable.

\section{Copyright}

(c) The Author(s) 2020.

\section{REFERENCES}

1. Volpe A, Panzarella T, Rendon RA, Haider MA, Kondylis FI, et al. The natural history of incidentally detected small renal masses. Cancer 2004;100:738-45.

2. Ljungberg B, Bensalah K, Canfield S, Dabestani S, Hofmann F, et al. EAU guidelines on renal cell carcinoma: 2014 update. Eur Urol 2015;67:913-24.

3. Johnson BA, Cadeddu JA. Current opinion in urology 2017: focal therapy of small renal lesions. Curr Opin Urol 2018;28:166-71. 